\title{
Efecto de cinco sustratos sobre índices de crecimiento de plantas de papaya (Carica papaya L.) bajo invernadero
}

\section{Effect of five substrates on growth indices of papaya (Carica papaya L.) plants under glasshouse conditions}

ARLETTE IVONNE GIL ${ }^{1}$

DIEGO MIRANDA²



\section{RESUMEN}

En este experimento se evaluó el efecto de cinco sustratos (turba rubia, suelo, mezcla de turba +cascarilla de arroz quemada, arena y el denominado "sustrato convencional") sobre el crecimiento de plantas de papaya variedad 'Maradol' y el híbrido 'Tainung-1', bajo condiciones de invernadero, ubicado en la Universidad Nacional de Colombia, Bogotá. Semanalmente se tomaron medidas de: área foliar y peso seco, durante el período comprendido entre la siembra hasta los 56 días después de emergencia (dde). Con esta información se calcularon los parámetros índice de área foliar (IAF), tasa de crecimiento relativo (TCR), tasa de crecimiento del cultivo (TCC) y tasa de asimilación neta (TAN). El sustrato más adecuado para ambos materiales fue la turba rubia (Klassman ${ }^{\circledR}$ ), debido a que provee las mejores características físico-químicas para el crecimiento y desarrollo de las plantas. Las plantas del híbrido 'Tainung-1' tuvieron un mejor comportamiento con respecto a la variedad 'Maradol'. Al final del ciclo, las plantas que crecieron en turba mostraron los valores más altos de TCC, con $1,34 \mathrm{~g} \cdot \mathrm{cm}^{-2} \cdot \mathrm{dí}^{-1}$ para 'Tainung-1' y $1,20 \mathrm{~g} \cdot \mathrm{cm}^{-2} \cdot \mathrm{d}^{-1}$ para 'Maradol'. Se observó que la adición de cascarilla o de gallinaza al sustrato tuvo un efecto negativo sobre el área foliar y el desarrollo del brote.

\footnotetext{
Profesora, Facultad de Ciencias Agropecuarias, Universidad de Cundinamarca, Fusagasugá (Colombia). arlettegil@hotmail.com

2 Profesor Asociado, Facultad de Agronomía, Universidad Nacional de Colombia, Bogotá (Colombia). dmirandal@unal.edu.co
} 
Palabras clave adicionales: variedad 'Maradol', híbrido 'Tainung-1', turba, tasas de crecimiento.

\section{ABSTRACT}

In this experiment there were evaluated the effects of five substrates (peat, soil, peat + burned rice husks mixture, sand and the so-called "conventional substrate") on the 'Maradol' papaya and the 'Tainung-1' hybrid plant growth, under greenhouse conditions, at the National University of Colombia, in Bogota. Leaf area and dry matter weight were weekly measured, from germination to 56 days after emergence (DAE) period. Leaf area index (LAI), relative growth rate (RGR), crop growth rate (CGR) and net assimilation rate (NAR) were calculated. Peat (Klasmann ${ }^{\circledR}$ ), was the most suitable substrate for both materials, because provided the best physical and chemical conditions for both growth and development. Thus, results showed that plants growing on both materials showed preference to peat-moss. The 'Tainung-1' hybrid showed a better behavior with respect to 'Maradol'. Finally, the plants growing on peat showed the highest CGR values with $1.34 \mathrm{~g} \cdot \mathrm{cm}^{-2}$.day ${ }^{1}$ for 'Tainung-1' and $1.20 \mathrm{~g} \cdot \mathrm{cm}^{-2} \cdot \mathrm{day}^{-1}$ for 'Maradol'. Furthermore, the supply of rice husks or hen manure affected negatively leaf area and shoot development.

Additional key words: 'Maradol' variety, 'Tainung-1' hybrid, peat, growth rates.

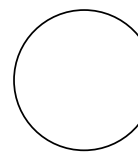

INTRODUCCIÓN

La síntesis continua de sustancias, desde pequeñas moléculas hasta grandes y complejas estructuras, constituye el crecimiento. Se le puede definir como el aumento de protoplasma o el incremento en peso seco o volumen irreversible que ocurre en un órgano o en la planta completa (Montaldi, 1995). El patrón de crecimiento de una generación se describe por medio de la curva sigmoide, que resulta de las tasas de crecimiento diferenciales durante su ciclo de vida (Hunt, 1990).

El análisis de crecimiento es una aproximación cuantitativa para entender el crecimiento de una planta o de una población de plantas bajo condiciones ambientales naturales o controladas. Es una técnica que utiliza modelos matemáticos para cuantificar la relación existente entre el crecimiento de una planta, la producción de materia seca y la expansión de área foliar, entre estos factores y una condición ambiental como la luz, el agua o los nutrientes (Clavijo, 1989). Las técnicas de análisis del crecimiento en plantas son una herramienta poderosa de comparación. El análisis de crecimiento tiene la gran ventaja de proveer medidas precisas del funcionamiento de la planta a través de intervalos de tiempo (Hunt, 1990).

La absorción de luz incidente sobre un cultivo depende de la amplitud de su superficie foliar. Una medida que expresa la capacidad de la estructura para interceptar la radiación solar es el índice de área foliar (IAF). Este índice representa la suma de la totalidad de las superficies de las hojas existentes en un área de suelo, expresadas ambas en las mismas unidades. Los cultivos de especies diferentes absorben distintas cantidades de fotones, aun con el mismo IAF. Este compor- 
tamiento se debe a la orientación de las hojas en el espacio (Montaldi, 1995).

Además del IAF, resulta útil considerar la disposición de las hojas en el dosel. Si todas las hojas están en posición aproximadamente horizontal, las hojas de la porción superior estarán expuestas a luz solar plena y la fotosíntesis en estas se verá sobresaturada, por lo que gran parte de la luz se desperdiciaría. Si las hojas se orientan en posición más o menos vertical, los rayos de luz serán más o menos paralelos a las superficies foliares, por lo que casi ninguna hoja se encontraría por encima del nivel de saturación (Salisbury y Ross, 1994).

Desde 1906 se trabajó en la búsqueda de un índice de eficiencia de un cultivar o de una especie como productor de materia seca, pero fisiólogos y matemáticos encontraron en 1920 un índice, que denominaron "tasa de asimilación neta" (TAN), que expresa el aumento de peso total de la planta, en función del área foliar y por unidad de tiempo (Montaldi, 1995).

Debido a la falta de estudios de análisis de crecimiento en papaya, se realizó esta investigación con el objeto de determinar el efecto de la utilización de cinco sustratos para la siembra de las plantas, sobre cuatro índices de crecimiento, índice de área foliar (IAF), tasa de crecimiento relativo (TCR), tasa de crecimiento del cultivo (TCC) y tasa de asimilación neta (TAN), con el fin de aportar un mayor conocimiento en esta especie de interés económico en el país, tomando una variedad (Maradol roja) y un híbrido (Tainung-1), y de los cuales este último presenta mayor área plantada y de alta demanda por los consumidores.

\section{MATERIALES Y MÉTODOS}

Se utilizó como material vegetal semillas de papaya, de la variedad 'Maradol roja' y el híbrido 'Tainung-1'. Inicialmente en el laboratorio, las semillas de papaya se colocaron en lotes de 50 unidades en cajas de petri, con tres repeticiones.
En cada caja de petri, se colocó papel toalla para manos, y se humedeció con agua destilada. Las semillas se colocaron encima, y se llevaron al germinador a una temperatura de $30^{\circ} \mathrm{C}$. Cuando se inició la germinación (protrusión radicular a través de la testa), las semillas se llevaron al invernadero, en la Facultad de Agronomía de la Universidad Nacional de Colombia, sede Bogotá, y se sembraron individualmente en materos que contenían cada sustrato a evaluar. Las condiciones de temperatura y humedad relativa diarias dentro del invernadero se registraron de 7 hasta los 56 días después de emergencia (dde), con promedios de $32,3^{\circ} \mathrm{C}$ y $81 \%$ diarios, respectivamente.

Durante este tiempo, las plantas se llevaban al Laboratorio de Fisiología de Cultivos de la Facultad de Agronomía, para proceder a medir su área foliar con el equipo planimeter Li-Cor (LI-3100, USA), posteriormente, se colocaron en la estufa de secado WTB Binder (South Korea), a $70^{\circ} \mathrm{C}$ de temperatura, durante $48 \mathrm{~h}$. Pasado este tiempo, se pesaban en la balanza Chyo Júpiter SDT-200, con el fin de determinar su peso seco.

Los sustratos utilizados fueron: turba alemana (Klasmann, KTS 1); turba+cascarilla de arroz quemada; suelo, proveniente de los invernaderos aledaños a la Facultad de Agronomía; arena de río lavada y el sustrato denominado "convencional", que consistió en 3 partes de suelo +1 parte de arena +1 parte de gallinaza. Todos los sustratos se esterilizaron en autoclave marca TKF, a $70^{\circ} \mathrm{C}$ durante $6 \mathrm{~h}$, a una humedad relativa del $90 \%$, con el propósito de eliminar patógenos que pudieran incidir sobre el desarrollo de las plantas.

Los muestreos se realizaron cada 7 días, tomando tres plantas por repetición y por tratamiento, con el fin de evaluar a cada una, su área foliar y peso seco, como medidas directas. Posteriormente, se procedió a determinar las medidas derivadas para realizar las gráficas correspondientes. Para el análisis estadístico, se realizaron análisis de varianza, contrastes ortogonales y pruebas de comparación múltiple de Tukey al 5\% de significancia. 
Las medidas directas obtenidas fueron área foliar y peso seco. A partir de las medidas directas, se utilizaron las fórmulas propuestas por Radford (1967), Evans (1972) y Hunt (1990), con el fin de calcular los índices de crecimiento (medidas derivadas), como sigue:

$\mathrm{IAF}=\mathrm{AF} / \mathrm{AS}$,

$\mathrm{TCR}=\left(\operatorname{lnW}_{2}-\operatorname{lnW_{1}}\right) /\left(\mathrm{T}_{2}-\mathrm{T}_{1}\right)$,

TCC: $(1 / \mathrm{AS}) *\left(\mathrm{~W}_{2}-\mathrm{W}_{1}\right) /\left(\mathrm{T}_{2}-\mathrm{T}_{1}\right)$,

$\mathrm{TAN}=\left(\mathrm{W}_{2}-\mathrm{W}_{1}\right) /\left(\mathrm{T}_{2}-\mathrm{T}_{1}\right) *\left(\ln \mathrm{AF}_{2}-\ln \mathrm{AF}_{1}\right) /\left(\mathrm{AF}_{2}-\mathrm{AF}_{1}\right)$,

Donde: IAF: índice de área foliar; AF: área foliar; AS: área de suelo; TCR: tasa de crecimiento relativo; W: peso seco; T: tiempo; TCC: tasa de crecimiento del cultivo; TAN: tasa de asimilación neta.

\section{RESULTADOS Y DISCUSIÓN}

\section{Índice de área foliar (IAF)}

Los resultados obtenidos muestran que el IAF, para los dos materiales de papaya evaluados, fue de tendencia ascendente, mostrando hasta los 28 dde un incremento no muy pronunciado, y a partir de esta fecha, el crecimiento fue de tendencia exponencial, para todos los sustratos evaluados (figura 1).
Las plantas de los dos materiales, mostraron mejor comportamiento en la turba, con el mayor AF al final del ciclo evaluado, 'Tainung-1' con un IAF final de 0,42 superó a 'Maradol' con 0,31. Esta tendencia se empezó a observar a partir de 28 dde, período hasta el cual las plantas dependen en su mayoría de las reservas del endospermo, y en menor grado, de los nutrimentos edáficos (Richards y Rowe, 1977).

Al incrementarse el área foliar, la respiración de mantenimiento (Rm) se incrementa, de esta manera se necesita más eficiencia de los sistemas fotosintético y radical para enviar los fotoasimilados y nutrientes hacia los vertederos (Schaffer et al., 1996). Después de la turba, las plantas sembradas en suelo presentaron valores de IAF final de 0,17\% para 'Tainung-1' y 0,16\% para 'Maradol'. Los tratamientos donde se obtuvo el menor desarrollo foliar fueron arena, turba+cascarilla y el tratamiento convencional. En estos sustratos fue común la restricción del desarrollo radical por impedimentos físicos y sanitarios. Esto coincide con varios autores que mencionan que las restricciones en el desarrollo de la raíz afectan el crecimiento. Características del suelo que limitan la penetración o que reducen las tasas de elongación, pueden reducir el desarrollo de la planta y el rendimiento. La reducción en la toma de agua

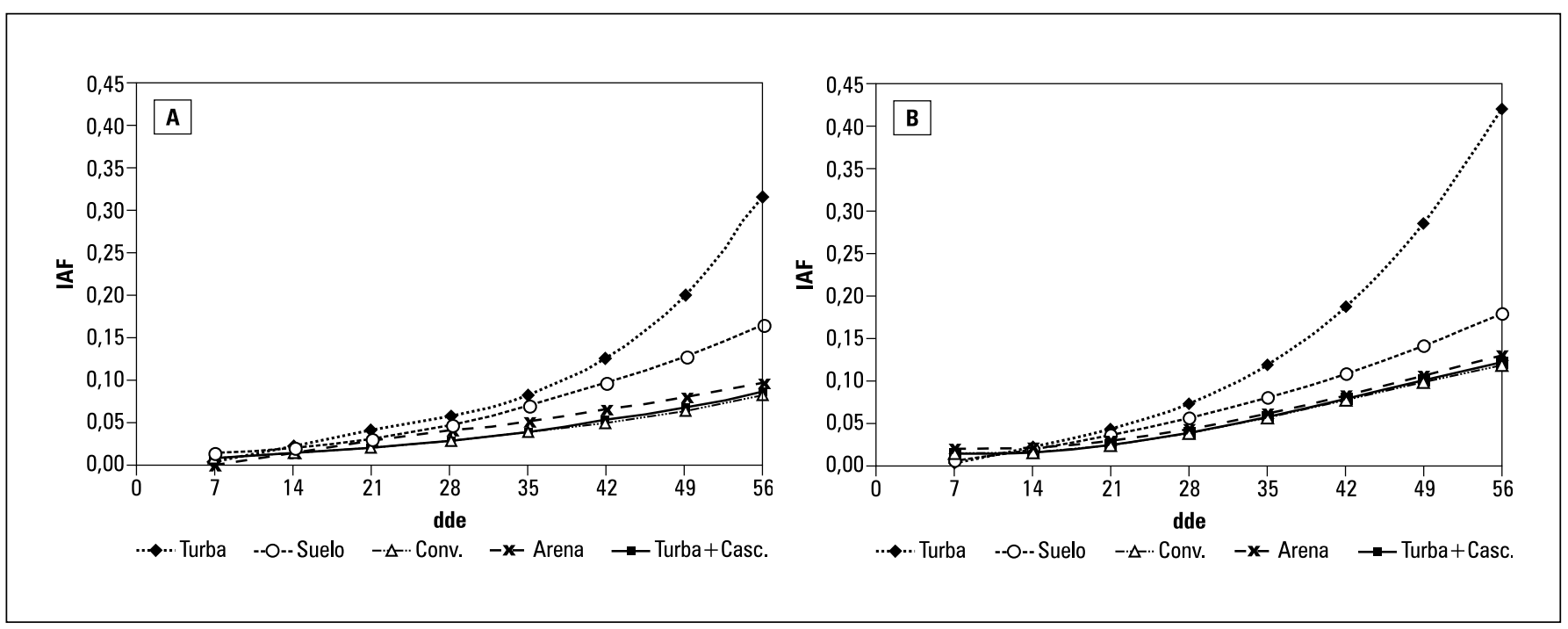

Figura 1. Tendencia del IAF para plantas de papaya, en la evaluación de cinco sustratos. A, 'Maradol'; B, 'Tainung-1'. 
y nutrientes depende, en gran parte, de la profundidad a la cual ocurre la zona de restricción (Unger y Kaspar, 1994). En plántulas de duraznero, la restricción de la raíz redujo el peso seco de la planta, el número de ápices de la raíz, el número de hojas, la iniciación de brotes, la extensión de la raíz, peso seco y longitud de la raíz, área foliar y toma de agua en 30-60\% (Richards y Rowe, 1977).

Como aseguran Abad et al. (2004), la arena por ser un sustrato inerte desde el punto de vista químico, por su capacidad de intercambio catiónico (CIC) nula y posible presencia de carbonatos pueden incrementar el $\mathrm{pH}$ del medio lo que ocasiona desordenes nutricionales, y afecta la toma de nutrientes y se ocasiona un desarrollo deficiente del brote, dando como consecuencia un menor desarrollo foliar. En el presente trabajo se presentó un IAF de 0,13\% para 'Tainung-1' y de 0,09\% para 'Maradol'. Para turba+cascarilla y el tratamiento convencional, tampoco se obtuvieron buenos resultados debido posiblemente a que en el primero, el contenido de taninos y posibles residuos de plaguicidas afectaron el desarrollo de la planta, obteniéndose un IAF de 0,12 para 'Tainung-1' y 0,08 para 'Maradol'. El tratamiento convencional, por su contenido de gallinaza como fuente de materia orgánica, afectó el desarrollo de las plantas, lo que se manifestó en un bajo IAF 0,11 para 'Tainung-1' y de 0,08 para 'Maradol'. La principal consecuencia del reducido crecimiento de la raíz es la consecuente dismi- nución del crecimiento del brote (Liu y Latimer, 1995; Hsu et al., 1996; Young et al., 1997; Hee Choi et al., 1997), Las ecuaciones de ajuste para las curvas de IAF se muestran en la tabla 1.

De acuerdo con la investigación realizada por Hernández et al. (1995) sobre el crecimiento del árbol de maraco (Theobroma bicolor H.B.K.) se concluyó que la acumulación de asimilados responde de manera directa a la expansión y duración del área foliar, coincidiendo en que esta es lenta durante los primeros estadios del desarrollo vegetativo, maximizándose durante los períodos de antesis y desarrollo del fruto.

Los valores altos del IAF en algunas ocasiones no están relacionados necesariamente con una mayor cantidad de fotosíntesis, ya que este índice es un concepto que representa para todo cultivo, un promedio de los estratos de follaje que están expandidos, situación que se ve afectada por el hecho de que las hojas no se despliegan sin dejar de encontrarse unas con otras, sino que lo hacen en diferentes ángulos que varían con la morfología de las especies y con las condiciones ambientales en que estén creciendo, de acuerdo con lo anotado por Barraza et al. (2004).

Según los contrastes ortogonales (tabla 2) sobre el comportamiento del área foliar en la evaluación de sustratos, se puede concluir que hubo diferencias significativas entre ambos materiales vegetales a partir de 14 dde, de manera que el

Tabla 1. Ecuaciones de ajuste para la tendencia de IAF en el tiempo, en la evaluación de sustratos.

\begin{tabular}{|l|l|l|l|l|}
\multicolumn{1}{|c|}{ Sustrato } & \multicolumn{1}{|c|}{ Maradol } & $R^{2}$ & Tainung-1 & $R^{2}$ \\
\hline Turba & $y=5 E-6 x^{3}-0,0003 x^{2}+0,0076 x-0,0382$ & 0,99 & $y=3 E-6 x^{3}-0,0001 x^{2}+0,0042 x-0,0208$ & 0,99 \\
\hline Suelo & $y=4 E-11 x^{3}+5 E-5 x^{2}-0,0002 x+0,0137$ & 0,99 & $y=2 E-7 x^{3}+2 E-5 x^{2}+0,0013 x-0,0043$ & 0,99 \\
\hline Convencional & $y=4 E-7 x^{3}-2 E-5 x^{2}+0,0012 x-1 E-04$ & 0,99 & $y=-9 E-7 x^{3}+0,0001 x^{2}-0,002 x+0,0238$ & 0,99 \\
\hline Arena & $y=4 E-7 x^{3}-4 E-5 x^{2}+0,0027 x-0,0167$ & 0,99 & $y=-6 E-7 x^{3}+9 E-5 x^{2}-0,0016 x+0,0273$ & 0,99 \\
\hline Turba + Cascarilla & $y=2 E-7 x^{3}+4 E-6 x^{2}+0,0007 x+0,0028$ & 0,99 & $y=-8 E-7 x^{3}+0,0001 x^{2}-0,0018 x+0,0221$ & 0,99 \\
\hline
\end{tabular}


Tabla 2. Valores $\mathbf{P}$ del análisis de varianza para el área foliar de las plantas de papaya, en la evaluación de sustratos.

\begin{tabular}{|c|c|c|c|c|c|c|c|c|c|c|c|c|c|c|c|c|c|}
\hline \multirow{2}{*}{ Fuente de variación } & \multicolumn{17}{|c|}{ Días después de emergencia } \\
\hline & $\mathrm{Gl}$ & 7 & & 14 & & 21 & & 28 & & 35 & & 42 & & 49 & & 56 & \\
\hline Tratamiento & 9 & 0,0001 & $* *$ & 0,0001 & $* *$ & 0,0001 & ** & 0,0001 & $* *$ & 0,0001 & ** & 0,0001 & ** & 0,0001 & ** & 0,0001 & $* *$ \\
\hline Variedad, V & 1 & 0,1871 & & 0,0001 & $* *$ & 0,0016 & ** & 0,0001 & $* *$ & 0,0001 & ** & 0,0001 & ** & 0,0001 & ** & 0,0001 & $* *$ \\
\hline Turba|Turba + Cascarilla & 1 & 0,0001 & ** & 0,0001 & $* *$ & 0,0001 & $* *$ & 0,0001 & $* *$ & 0,0001 & ** & 0,0001 & ** & 0,0001 & ** & 0,0001 & $* *$ \\
\hline Anterior $\times \mathrm{V}$ & 1 & 0,6303 & & 0,5022 & & 0,0119 & $*$ & 0,0231 & * & 0,0001 & ** & 0,0001 & ** & 0,0001 & ** & 0,0001 & $* *$ \\
\hline Turba si|no & 1 & 0,1073 & & 0,5452 & & 0,1843 & & 0,1635 & & 0,0001 & ** & 0,0001 & ** & 0,0001 & ** & 0,0001 & $* *$ \\
\hline Anterior $\times \mathrm{V}$ & 1 & 0,0019 & $* *$ & 0,1811 & & 0,2274 & & 0,0063 & $* *$ & 0,0001 & $* *$ & 0,0001 & ** & 0,0001 & ** & 0,0001 & $* *$ \\
\hline Suelo 0 arena & 1 & 0,1076 & & 0,0010 & $* *$ & 0,0010 & ** & 0,0001 & $* *$ & 0,0001 & $* *$ & 0,0001 & $* *$ & 0,0001 & $* *$ & 0,0001 & $* *$ \\
\hline Anterior $\times \mathrm{V}$ & 1 & 0,1184 & & 0,0001 & $* *$ & 0,6316 & & 0,0238 & * & 0,7252 & & 0,3990 & & 0,0053 & ** & 0,0099 & $* *$ \\
\hline Convencional | resto & 1 & 0,0001 & ** & 0,0001 & $* *$ & 0,0001 & $* *$ & 0,0001 & $* *$ & 0,0001 & ** & 0,0001 & $* *$ & 0,0001 & ** & 0,0001 & $* *$ \\
\hline Anterior x V & 1 & 0,1466 & & 0,2339 & & 0,7043 & & 0,9578 & & 0,4107 & & 0,5412 & & 0,1252 & & 0,0516 & \\
\hline Error muestral & 20 & & & & & & & & & & & & & & & & \\
\hline Error experimental & 60 & & & & & & & & & & & & & & & & \\
\hline
\end{tabular}

** , * Diferencia significativa, según la prueba de Tukey $P<0,01$ y $P<0,05$ respectivamente.

híbrido 'Tainung-1' mostró mayor desarrollo foliar. Entre los tratamientos de turba, se presentaron diferencias estadísticas, ya que se observó que la adición de cascarilla al sustrato tuvo un efecto negativo sobre el área foliar (González et al., 2006). Entre el tratamiento convencional y los demás, se presentaron diferencias significativas para todas las fechas de muestreo, y resultó ser el menos adecuado entre todo el conjunto de sustratos evaluados Abad et al. (2004).

\section{Tasa de crecimiento relativo (TCR)}

La TCR depende de la acumulación de materia seca en el tiempo. Su tendencia fue decreciente, tanto para 'Maradol' como para 'Tainung-1', tal como se observa en la figura 2.

El decrecimiento de la TCR es de manera abrupta en su pendiente hasta 28 dde, debido a que en este momento la planta es pequeña y está gastando sus reservas endospérmicas principalmente, con lo cual se presenta un gasto alto de energía debido a la respiración de crecimiento $(\mathrm{Rc})$, y adicionalmente empieza a extraer los nutrientes disponibles del sustrato en el cual está sembrada (Abad et al., 2004). Como resultado, el metabolismo se acelera con el fin de producir los esqueletos carbonados para su crecimiento (Arp, 1991, Salisbury, y Ross, 1994). A partir de esta fecha la pendiente se suaviza debido a que se incrementa la materia seca, con lo cual la Rm se hace mayor que en la etapa anterior, y por tanto disminuye la TCR. Las ecuaciones de ajuste se muestran en la tabla 3.

Esta tendencia decreciente de la TCR coincidió con la reportada por Mazorra et al. (2003), en un estudio realizado en uchuva (Physalis peruviana), con lo cual se concluye que la TCR es un parámetro de crecimiento muy sensible a las condiciones climáticas donde se desarrolló el cultivo. De igual forma, Barraza et al. (2004) en una investigación realizada en tomate (Lycopersicon esculentum), reportan que existe correlación entre el proceso de crecimiento y desarrollo de diversas partes de la planta, que puede explicarse en términos del suministro y la demanda. Cuanto más activo sea el crecimiento de una parte de la planta, tanto más demandará materiales disponibles, y tanto más se restringirá el crecimiento en otras partes. Debido a la restricción del sistema radical podría haber deficiente suplemento hídrico (Hameed et 

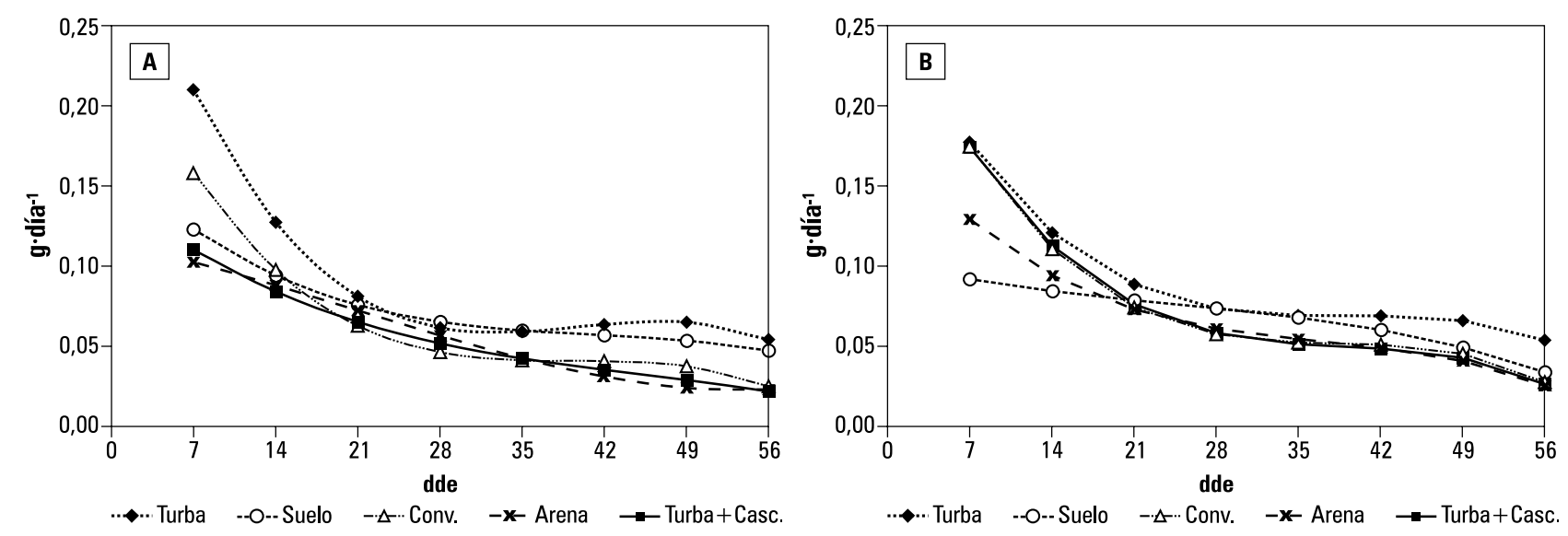

Figura 2. Tendencia de la TCR para plantas de papaya, en la evaluación de cinco sustratos. A, 'Maradol'; B, 'Tainung-1'.

al., 1987; Tschaplinski y Blake, 1985; Ran et al., 1992) y de nutrientes (Hanson et al., 1987; Dubik et al., 1990; Rieger y Marra, 1994) hacia el brote. Además una reducción en la raíz "fuente" como resultado de la restricción de la raíz puede causar acumulación de carbohidratos en la hoja, resultando en una respuesta inhibitoria de la fotosíntesis (Arp, 1991; Schaffer et al., 1996).

Para los dos materiales evaluados, se observó que la turba fue el sustrato más adecuado, pues en él, las plantas presentaron los valores más altos de TCR al final del ciclo de evaluación, de 0,05 g.día ${ }^{-1}$ para ambos cultivares, seguido por las plantas en el suelo, con 0,03 g.día ${ }^{-1}$ para 'Tainung-1' y 0,04 g.día ${ }^{-1}$ para 'Maradol'. Las turbas presentan un efecto estimulador sobre el crecimiento y desarrollo vegetal, lo que se ha atribuido a la presencia de activadores del crecimiento (Abad et al., 2004). Las plantas con menores valores de TCR se encontraron en arena, con 0,02 g.día ${ }^{1}$ para 'Tainung-1' y 0,02 g.día-1 para 'Maradol', debido a que, si bien esta permite un mejor desarrollo de las raíces, por el hecho de ser estéril, las plantas de papaya debieron utilizar sus reservas endospérmicas, y a partir de los 28 dde incrementar su eficiencia para la acumulación de materia seca, por tanto las tasas de respiración se debieron incrementar. Los sustratos menos apropiados fueron turba+cascarilla, donde las TCR fueron 0,02 g.día ${ }^{-1}$ para 'Tainung-1' y 0,02 para 'Maradol', y el sustrato convencional, con 0,02 g.día ${ }^{-1}$ para ambos cultivares. La mezcla de sustratos hacen que algunas de sus propiedades como retención de humedad, capacidad de aireación, la inactividad química y biológica entre otras, se alteren produciéndose diferentes efectos sobre el crecimiento de las raíces y del brote de las plantas (Calderón y Cevallos, 2001).

Al analizar los contrastes ortogonales realizados al peso seco en el tiempo, se presentaron diferencias significativas entre la variedad y el híbrido a partir de los 21 dde. De acuerdo con los tratamientos, se presentaron diferencias estadísticas entre la acumulación de masa seca por las plantas establecidas en turba y turba+cascarilla a partir de los 14 dde, en este último la adición de cascarilla produjo un efecto negativo sobre la acumulación de masa seca, posiblemente ocasionado por un menor número de raíces, con desarrollo incipiente y pérdida de raíces, posiblemente por residuos existentes en la cascarilla. También se presentaron diferencias significativas entre el 
Tabla 3. Ecuaciones de ajuste para la tendencia de TCR en el tiempo, en la evaluación de sustratos.

\begin{tabular}{|l|l|l|l|l|}
\multicolumn{1}{|c|}{ Sustrato } & \multicolumn{1}{|c|}{ Maradol } & $R^{2}$ & \multicolumn{1}{c|}{ Tainung-1 } & $R^{2}$ \\
\hline Turba & $y=-5 E-6 x^{3}+0,0006 x^{2}-0,0222 x+0,339$ & 0,92 & $y=-3 E-6 x^{3}+0,0004 x^{2}-0,0149 x+0,2642$ & 0,96 \\
\hline Suelo & $y=-1 E-6 x^{3}+0,0002 x^{2}-0,007 x+0,1647$ & 0,99 & $y=-6 E-7 x^{3}+4 E-5 x^{2}-0,0018 x+0,1025$ & 0,98 \\
\hline Convencional & $y=-3 E-6 x^{3}+0,0004 x^{2}-0,0159 x+0,2509$ & 0,95 & $y=-4 E-6 x^{3}+0,0004 x^{2}-0,0171 x+0,2741$ & 0,98 \\
\hline Arena & $y=7 E-7 x^{3}-4 E-5 x^{2}-0,0014 x+0,1139$ & 0,99 & $y=-2 E-6 x^{3}+0,0002 x^{2}-0,009 x+0,1818$ & 0,98 \\
\hline Turba + Cascarilla & $y=-8 E-7 x^{3}+0,0001 x^{2}-0,0057 x+0,1448$ & 0,94 & $y=-3 E-6 x^{3}+0,0004 x^{2}-0,0162 x+0,2689$ & 0,99 \\
\hline
\end{tabular}

sustrato turba y los demás. Entre el tratamiento convencional y el resto hubo diferencias estadísticas para todos los muestreos.

\section{Tasa de crecimiento del cultivo (TCC)}

La TCC mostró una tendencia creciente en el tiempo (figura 3), para 'Maradol' y 'Tainung-1', debido a que esta tasa representa la ganancia en peso de una comunidad de plantas por unidad de área de suelo por unidad de tiempo. Las ecuaciones de ajuste de las curvas se muestran en la tabla 4.

Para los dos materiales se observó que en general hasta 28 dde, el crecimiento del cultivo fue lento debido a que existen pocas hojas en relación con el área de suelo. A partir de este momento, se incrementó el número de hojas y el área foliar, en la misma área de suelo, por tanto, se aumentan los valores de TCC, lo que se observa en la curva como un aumento exponencial.

Al final del ciclo las plantas que crecieron en turba mostraron los valores más altos de TCC, con 1,34 $\mathrm{g} \cdot \mathrm{cm}^{-2} \cdot \mathrm{día}^{-1}$ para 'Tainung-1' y $1,20 \mathrm{~g} \cdot \mathrm{cm}^{-2} \cdot \mathrm{día}^{-1}$ para 'Maradol', seguidas de aquellas sembradas en suelo, con valores de $0,74 \mathrm{~g} \cdot \mathrm{cm}^{-2} \cdot \mathrm{dí}^{-1}$ para 'Tainung-1' y de $0,71 \mathrm{~g} \cdot \mathrm{cm}^{-2} \cdot \mathrm{dí}^{-1}$ para 'Maradol'. Las plantas sembradas en arena, turba+cascarilla y convencional, dieron como resultado los valores más bajos de TCC. El sustrato arena no suminis- tró los nutrientes necesarios para el crecimiento, con TCC de $0,28 \mathrm{~g} \cdot \mathrm{cm}^{-2}$. día ${ }^{-1}$ para 'Tainung-1' y $0,15 \mathrm{~g} \cdot \mathrm{cm}^{-2} \cdot$ día $^{-1}$ para 'Maradol'; el sustrato de turba+cascarilla quemada afectó el incremento de área foliar debido a la presencia de taninos y posibles residuos de herbicida en la cascarilla, con valores de $0,26 \mathrm{~g} \cdot \mathrm{cm}^{-2} \cdot$ día $^{-1}$ para 'Tainung1 ' y de $0,15 \mathrm{~g} \cdot \mathrm{cm}^{-2} \cdot \mathrm{dí}^{-1}$ para 'Maradol', y el convencional por contener gallinaza como fuente de materia orgánica afectó la superficie foliar en tamaño, con 0,25 g. $\mathrm{cm}^{-2}$.día ${ }^{-1}$ para 'Tainung-1' y $0,14 \mathrm{~g} \cdot \mathrm{cm}^{2} \cdot \mathrm{día}^{-1}$ para 'Maradol'. A pesar de ello, las plantas del híbrido sembradas en estos tres sustratos presentaron las mayores TCC en relación con las de la variedad, lo cual indica que ellas superaron los efectos negativos de los sustratos debidos en parte a la heterosis y su expresión como vigor híbrido.

Estos resultados coinciden con los reportados por Barraza et al. (2004) en tomate (Lycopersicon esculentum), en donde la TCC aumentó rápidamente hasta alcanzar un máximo, y posteriormente este índice de crecimiento decreció en forma acelerada. Los mayores valores de TCC reflejan mayor ganancia en peso de materia seca por unidad de superficie de suelo y de tiempo. Según Rodríguez et al. (2003), el potencial genético de las plantas y las condiciones ambientales afectan la velocidad del crecimiento vegetal. También se observó que a partir del día 28 dde las plantas empiezan 
a depender de su capacidad fotosintética, para incrementar sus tasas de crecimiento en forma independiente de las reservas aún existentes en la semilla.

\section{Tasa de asimilación neta (TAN)}

La TAN es un índice dependiente del peso seco y el área foliar, es decir, de los procesos de fotosíntesis y respiración, de acuerdo con Montaldi
(1995). Con base en esta premisa y según los resultados obtenidos, se observó que la tendencia de la TAN fue decreciente en el tiempo tanto para 'Maradol' como para 'Tainung-1', en todos los sustratos evaluados. La eficiencia de las plantas como sistema asimilatorio se observó al final del ciclo de evaluación, es decir que las plantas de los tratamientos con los mayores valores de TAN fueron las más eficientes. Hasta 28 dde la TAN presentó un descenso pronunciado, y después de

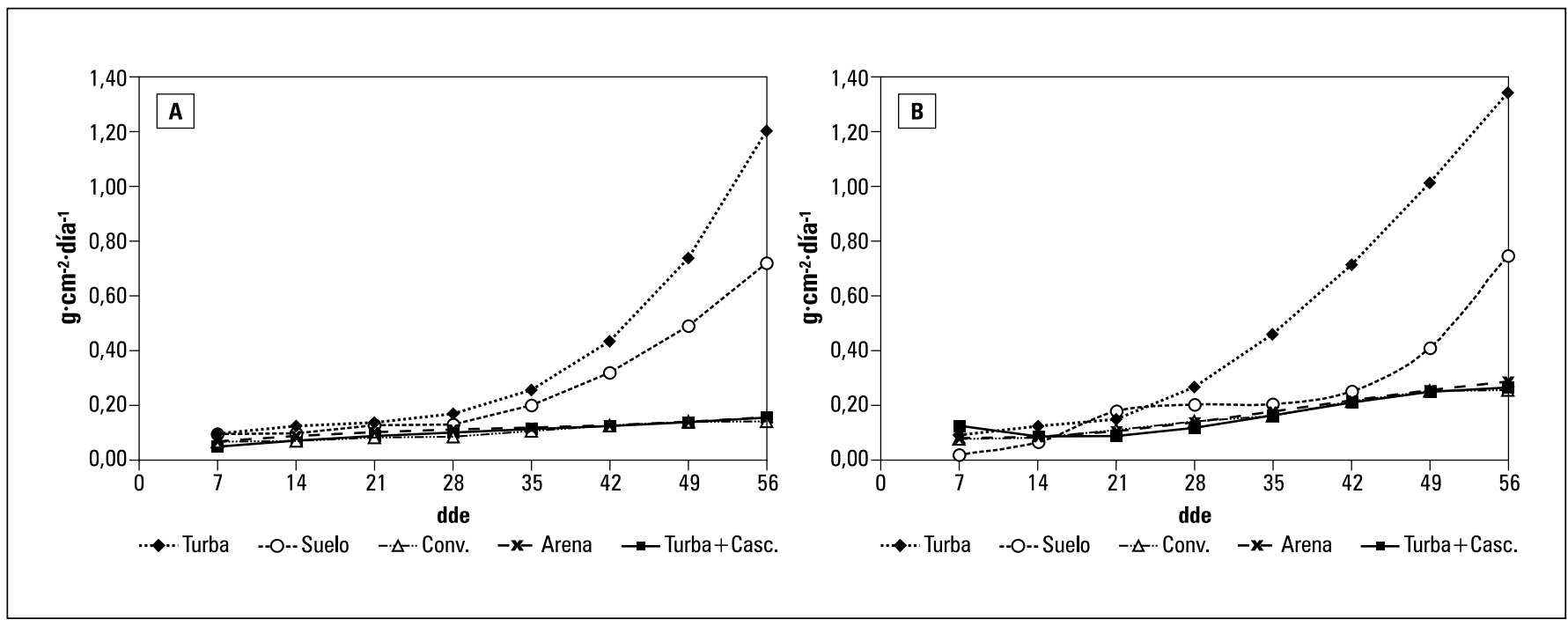

Figura 3. Tendencia de la TCC para plantas de papaya, en la evaluación de cinco sustratos. A, 'Maradol'; B, 'Tainung-1'.

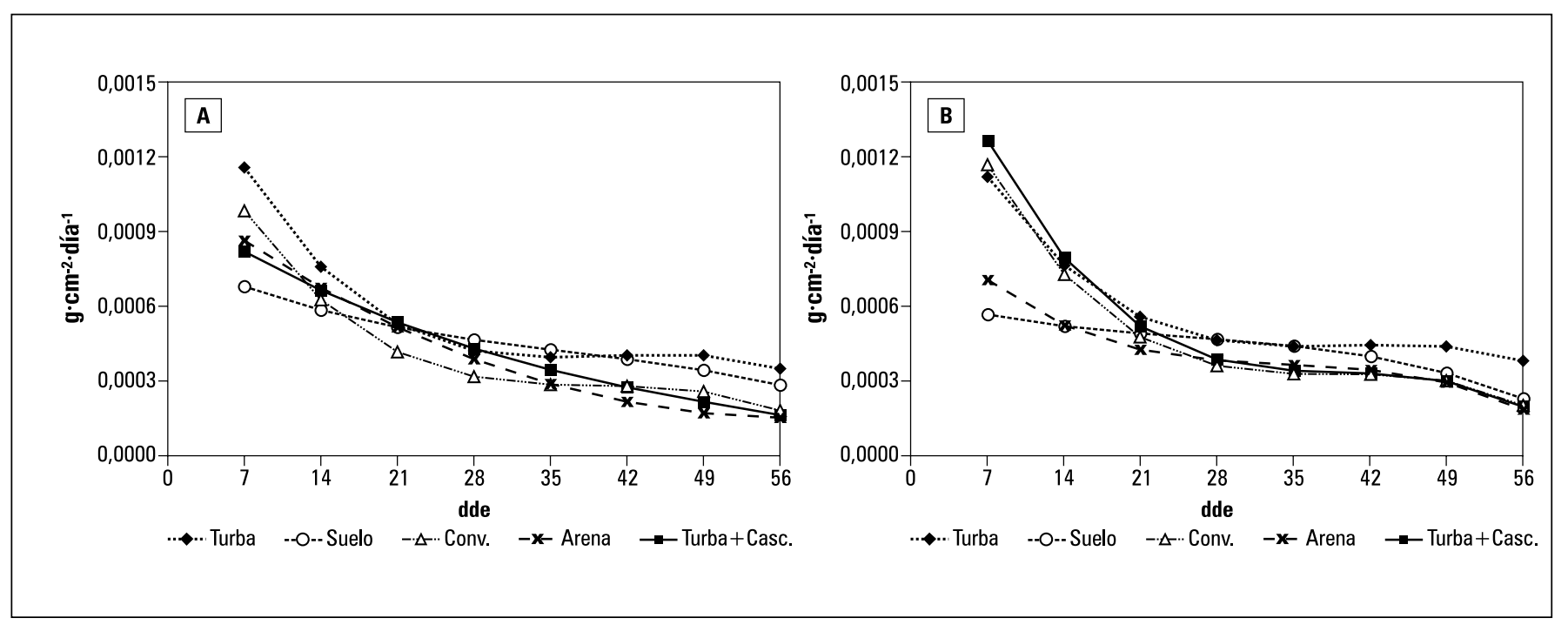

Figura 4. Tendencia de la TAN para plantas de papaya, en la evaluación de cinco sustratos. A, 'Maradol'; B, 'Tainung-1'. 
esta fecha la tendencia fue a estabilizarse (figura 4). Las correspondientes ecuaciones de ajuste se muestran en la tabla 5 .

Para las plantas sembradas en turba la TAN descendió drásticamente hasta los 28 dde, período donde termina la fase de endurecimiento de las plántulas, como lo demostró Baiyeri (2006). Debido a que su metabolismo es más rápido, quizás debido a que la toma de los nutrientes contenidos en el sustrato colaboran en este aspecto, de este modo su Rc es mayor. Los valores finales fueron de $0,00038 \mathrm{~g} \cdot \mathrm{cm}^{-2} \cdot \mathrm{dí}^{-1}$ para 'Tainung-1' y de $0,00035 \mathrm{~g} \cdot \mathrm{cm}^{-2} \cdot \mathrm{dia}^{-1}$ para 'Maradol'. Para el suelo, la pendiente descendió suavemente, lo que quiere decir que la Rc no fue tan alta como en turba, debido a que el suelo no suministró suficientes nutrimentos para el crecimiento de las plantas. A partir de esta fecha el descenso fue más suave debido a que la Rm aumentó, con el fin de sostener las estructuras ya formadas y presentó valores finales de $0,00023 \mathrm{~g} \cdot \mathrm{cm}^{-2} \cdot \mathrm{dí}^{-1}$ para 'Tainung-1' y de $0,00028 \mathrm{~g} \cdot \mathrm{cm}^{-2} \cdot$ día $^{-1}$ para 'Maradol'. Las plantas que crecieron en los otros tres sustratos obtuvieron los valores más bajos de TAN al final del ciclo de evaluación, indicando que la eficiencia fue baja y su crecimiento se vio afectado debido a que estaban invirtiendo mucho más en Rm que en Rc. La TAN de las plantas establecidas en arena fue de $0,00019 \mathrm{~g} \cdot \mathrm{cm}^{-2} \cdot \mathrm{día}^{-1}$ para 'Tainung-1' y 0,0015 g.cm ${ }^{-2} \cdot \mathrm{dí}^{-1}$ para 'Maradol'. En turba+cascarilla, de $0,00020 \mathrm{~g} \cdot \mathrm{cm}^{-}$ 2.día ${ }^{-1}$ para 'Tainung-1' y 0,00016 g.cm ${ }^{-2} \cdot \mathrm{día}^{-1}$ para 'Maradol', y en sustrato convencional, de $0,00020 \mathrm{~g} \cdot \mathrm{cm}^{-2} \cdot \mathrm{día}^{-1}$ para 'Tainung-1' y 0,00018 $\mathrm{g} \cdot \mathrm{cm}^{-2} \cdot \mathrm{dí}^{-1}$ para 'Maradol'.

Tabla 4. Ecuaciones de ajuste para la tendencia de TCC en el tiempo, en la evaluación de sustratos.

\begin{tabular}{|c|c|c|c|c|}
\hline Sustrato & Maradol & $R^{2}$ & Tainung-1 & $R^{2}$ \\
\hline Turba & $y=2 E-5 x^{3}-0,0009 x^{2}+0,0166 x+0,0178$ & 0,99 & $y=-2 E-6 x^{3}+0,0008 x^{2}-0,0162 x+0,1815$ & 0,99 \\
\hline Suelo & $y=6 E-6 x^{3}-0,0002 x^{2}+0,0024 x+0,0874$ & 0,99 & $y=2 E-5 x^{3}-0,0017 x^{2}+0,0462 x-0,2502$ & 0,95 \\
\hline Convencional & $y=-1 E-6 x^{3}+0,0001 x^{2}-0,0026 x+0,0811$ & 0,98 & $y=-3 E-6 x^{3}+0,0003 x^{2}-0,0047 x+0,098$ & 0,98 \\
\hline Arena & $y=9 E-7 x^{3}-9 E-5 x^{2}+0,0048 x+0,0193$ & 0,97 & $y=-2 E-6 x^{3}+0,0003 x^{2}-0,004 x+0,0975$ & 0,99 \\
\hline Turba + Cascarilla & $y=9 E-7 x^{3}-9 E-5 x^{2}+0,0048 x+0,0193$ & 0,95 & $y=-6 E-6 x^{3}+0,0007 x^{2}-0,0175 x+0,2192$ & 0,98 \\
\hline
\end{tabular}

Tabla 5. Ecuaciones de ajuste para la tendencia de la TAN en el tiempo, para el análisis de sustratos.

\begin{tabular}{|l|l|l|l|l|}
\multicolumn{1}{|c|}{ Sustrato } & \multicolumn{1}{|c|}{ Maradol } & $R^{2}$ & Tainung-1 \\
\hline Turba & $y=-2 E-8 x^{3}+3 E-6 x^{2}-0,0001 x+0,0018$ & 0,97 & $y=-2 E-8 x^{3}+2 E-6 x^{2}-9 E-5 x+0,0017$ & 0,96 \\
\hline Suelo & $y=-4 E-9 x^{3}+4 E-7 x^{2}-2 E-5 x+0,0008$ & 0,95 & $y=-5 E-9 x^{3}+4 E-7 x^{2}-1 E-5 x+0,0006$ & 0,98 \\
\hline Convencional & $y=-2 E-8 x^{3}+2 E-6 x^{2}-9 E-5 x+0,0015$ & 0,95 & $y=-3 E-8 x^{3}+3 E-6 x^{2}-0,0001 x+0,0018$ & 0,98 \\
\hline Arena & $y=-4 E-10 x^{3}+3 E-7 x^{2}-3 E-5 x+0,0011$ & 0,99 & $y=-1 E-8 x^{3}+1 E-6 x^{2}-5 E-05 x+0,001$ & 0,99 \\
\hline Turba + Cascarilla & $y=-2 E-9 x^{3}+4 E-7 x^{2}-3 E-5 x+0,001$ & 0,96 & $y=-3 E-8 x^{3}+3 E-6 x^{2}-0,0001 x+0,002$ & 0,99 \\
\hline
\end{tabular}


Según Barraza et al. (2004), los mayores valores de TAN indican mayor ganancia de materia seca por unidad de tejido asimilatorio y por unidad de tiempo, lo cual indica una mayor eficiencia fotosintética promedio. La disminución en la velocidad de asimilación neta de fotoasimilados, es un reflejo de que en la medida en que se producen más hojas, las hojas inferiores van quedando sombreadas y sus tasas fotosintéticas disminuyen en relación directa con la disponibilidad de radiación solar. Trabajos similares en variedades de papaya reportados por Baiyeri (2006) demostraron que para el desarrollo de las plántulas el efecto de la intensidad de luz, la temperatura y en parte el autosombreamiento foliar explican en $40 \%$ las diferencias obtenidas en la eficiencia fotosintética de las plantas.

\section{CONCLUSIONES}

A partir de los resultados obtenidos, se puede concluir que la turba es el mejor sustrato para el sostenimiento de las plantas de papaya en los primeros estadios de desarrollo debido a que ofrece las mejores características físicas y químicas, permite un buen desarrollo, de las raíces, no presenta compactación cuando está húmeda y presenta disponibilidad de nutrientes para la planta. Como segunda opción se puede utilizar el suelo, teniendo en cuenta de realizar los correctivos necesarios en presiembra.

No se recomienda adicionar cascarilla quemada a la turba, ni utilizar gallinaza como fuente de materia orgánica (tratamiento convencional), pues presentaron inconvenientes para el desarrollo radical, como tampoco utilizar arena únicamente como sustrato, debido a que estos no ofrecen los requerimientos nutricionales para el cultivo durante las primeras etapas de su crecimiento.

Bajo las condiciones del experimento, las plantas del híbrido 'Tainung-1' mostraron un comportamiento superior que las de la variedad 'Maradol', de acuerdo con los índices de crecimiento evaluados (IAF, TCR, TCC y TAN), lo cual es debido en parte a la heterosis o vigor híbrido.

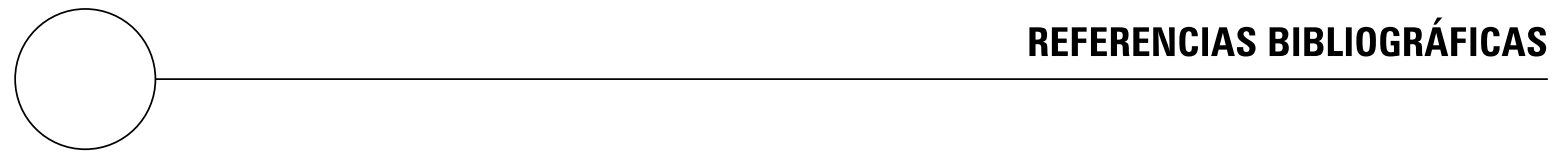

Abad, M.; P. Noguera y C. Carrión. 2004. Los sustratos en los cultivos sin suelo. pp. 113-158. En: Urrestarazu, M. (e.d.) Tratado de cultivos sin suelo. Ediciones Mundi-Prensa, Madrid.

Arp, W.J. 1991. Effects of source-sink relations on photosynthetic acclimation to elevated $\mathrm{CO}_{2}$. Plant Cell Environ. 14, 869-875.

Barraza, F.; G. Fischer y C. Cardona. 2004. Estudio del proceso de crecimiento del cultivo del tomate (Lycopersicon esculentum Mill.) en el valle del Sinú medio, Colombia. Agron. Colomb. 22(1), 81-90.

Baiyeri, K.P. 2006. Seedling emergence and growth of pawpaw (Carica papaya) grown under different coloured shade polyethylene. Institute of Agrophysics, Polish Academy of Sciences 20, 77-84.

Calderón, S. y F. Cevallos. 2001. "los sustratos". En: http:// www.drcalderonlabs.com/Publicaciones/Los_Sustratos.htm; consulta: junio de 2004.
Clavijo, J. 1989. Análisis del crecimiento en malezas. Revista Comalfi 26, 12-16.

Dubik, S.P.; D.P. Krizek y D.P. Stimart, 1990. Influence of root zone restriction on mineral element concentration, water potential, chlorophyll concentration, and partitioning of assimilate in spreading euonymus (E. kiautschovica Loes. Sieboldiana). J. Plant Nutr. 13, 677-699.

Evans, G.C. 1972. The cuantitative analysis of plant growth. Studies in ecology. Vol.1. Blackwell Scientific Publication, Londres. pp. 45-68.

González, C.; M.F. Quintero y V. Meneses. 2006. Determinación de algunas propiedades hidrofísicas de $\mathrm{k}$, los sustratos utilizados en cultivos de flores en la sabana de Bogotá. pp. 427-450. En: Flórez, V.; A. Fernández; D. Miranda; B. Chaves y M. Guzmán (eds.). Avances sobre fertirriego en la floricultura colombiana. Unibiblos, Bogotá. 
Hameed, M.A.; J.B. Reid y R.N. Rowe. 1987. Root confinement and its effects on the water relations, growth and assimilate partitioning of tomato (Lycopersicun esculentum Mill). Ann. Bot. 59, 685-692.

Hanson, P.J.; R.K. Dixon y R.E. Dickenson. 1987. Effect of container size and shape on the growth of northern red oak seedlings. HortScience 22, 1293-1295.

Hee Choi, J.; G. Chae Chung; S. Ryong Suh; J. Ah Yu; J. Hoon Sung y K. Ju. 1997. Suppression of calcium transport to shoots by root restriction in tomato plants. Plant Cell Physiol. 38, 495-498.

Hernández, M.S.; A. Casas; O. Martínez y J.A. Galvis. 1995. Análisis y estimación de parámetros e índices de crecimiento delárbol de maraco (Theobroma bicolor H.B.K.) a primera floración. Agron. Colomb. 12(1), 182-191.

Hsu, Y.M.; M.J. Tseng y C.H. Lin. 1996. Container volume effects growth and development of wax-apple. HortScience 31, 1139-1142.

Hunt, R. 1990. Basic growth analysis: plant growth analysis for beginners. Unwin Hyman, Londres. 112p.

Liu, A.; y J.G. Latimer. 1995. Water relations and abscisic acid levels of watermelon as affected by rooting volume restriction. J. Exp. Bot. 46, 1011-1015.

Mazorra, M.; A. Quintana; D. Miranda; G. Fischer y B. Chaves. 2003. Análisis sobre el desarrollo y la madurez fisiológica del fruto de la uchuva (Physalis peruviana L.) en la zona de Sumapaz (Cundinamarca). Agron. Colomb. 21(3), 175-189.

Montaldi, E. 1995. Principios de fisiología vegetal. Ediciones Sur, La Plata, Argentina 298 p.

Radford, P.J. 1967. Growth analysis formulae. Their use and abuse. Crop Sci. 7, 171-175.
Ran, Y.; B. Bar-Yosef y A. Erez. 1992. Root volume influence on dry matter production and partitioning as related to nitrogen and water uptake rates by peach tree. J. Plant Nutr. 15, 713-726.

Richards, D. y R.N. Rowe. 1977. Effects of root restriction, root pruning, and 6-benzylaminopurine on the growth of peach seedlings. Ann. Bot. 41, 729-740.

Rieger, M. y F. Marra. 1994. Responses of young peach trees to root confinement. J. Amer. Soc. Hort. Sci. $119,223-228$.

Rodríguez, L.; G. Corchuelo y C. Ñustez. 2003. Influencia del espaciamiento entre plantas sobre la morfología y el crecimiento de la papa (Solanum tuberosum L. cv. Parda pastusa) bajo dos ambientes contrastantes. Agron. Colomb. 21(3), 210-219.

Salisbury, F.B. y C.W. Ross. 1994. Fisiología vegetal. Grupo Editorial Iberoamérica, México D.F. 760 p.

Schaffer, B.C.; C. Searle; A.W. Whiley y R.J. Nissen. 1996. Effects of atmospheric $\mathrm{CO}_{2}$ enrichment and root restriction on leaf gas exchange and growth of banana (Musa). Physiol. Plant. 97, 685-693.

Tschaplinski, T.J. y T.J. Blake. 1985. Effects of root restriction on growth correlations, water relations and senescence of alder seedlings. Physiol. Plant. $64,167-176$.

Unger, P.W. y T. Kaspar. 1994. Soil compaction and root growth: a review. Agron. J. 86, 759-766.

Young, I.M.; K. Montagu y J.A.G. Conroy, 1997. Mechanical impedance of root growth directly reduces leaf elongation rates of cereals. New Phytol. 135, 613-619. 